\title{
NATURAL DUALITY VIA A FINITE SET OF RELATIONS
}

\author{
LÁSZLÓ ZÁDORI
}

\begin{abstract}
We present a duality theorem. We give a necessary and sufficient condition for any set of algebraic relations to entail the set of all algebraic relations in Davey and Werner's sense. The main result of the paper states that for a finite algebra a finite set of algebraic relations yields a duality if and only if the set of all algebraic relations can be obtained from it by using four types of relational constructs. Finally, we prove that a finite algebra admits a natural duality if and only if the algebra has a near unanimity term operation, provided that the algebra possesses certain $2 k$-ary term operations for some $k$. This is a generalisation of a theorem of Davey, Heindorf and McKenzie.
\end{abstract}

\section{INTRODUCTION}

Let $\underline{P}$ be a finite algebra on the underlying set $P$. We define $\mathbf{P}$ to be the relational topological structure $P$ equipped with all subalgebras of finite powers (algebraic relations) of $\underline{P}$ and the discrete topology. For an arbitrary power $P^{S}$ any subset $X \subseteq P^{S}$ inherits a structure from $P$ via restricting the product structure to $X$. This structured subset is denoted by $\mathbf{X}$ and called a substructure. If $\mathbf{X}$ is a closed subset in the product topology on $P^{S}$ then we call it a closed substructure. A morphism between two closed substructures means a structure preserving map, that is, a continuous map which preserves the given relations on its domain. For an arbitrary set $X \subseteq P^{S}$ let $\neg X$ denote the subalgebra of $\underline{P}^{X}$ generated by $\pi_{s} \mid x, s \in S$, where $\pi_{0}: P^{S} \rightarrow P$ is the $s$-th projection for each $s \in S$. A subset $X$ of $P^{S}$ is called hom-closed, if every homomorphism $f: \neg X \rightarrow \underline{P}$ is the $x$-th projection for some $x \in X$. This definition of hom-closed sets is equivalent to the usual one given in [2] and [3, Theorem 2.1]. A subset $X$ of $P^{S}$ is term-closed if for every element $a \in P^{S} \backslash X$ there exist two $S$-ary $\underline{P}$-term operations agreeing on every element of $X$ and differing on $a$. It is easy to show, see [2, Lemma 2.15], that $X \subseteq P^{S}$ is hom-closed if and only if it is term-closed. Moreover, every term-closed set $X \subseteq P^{S}$ is closed in the product topology on $P^{S}$. For every set $X \subseteq P^{S}$ the smallest term-closed subset containing $X$ in $P^{S}$ is called the term-closure of $X$ and is denoted by $X^{\prime}$.

Received 25th July, 1994

Research partially supported by Hungarian National Foundation for Research under grant no. 1903.

Copyright Clearance Centre, Inc. Serial-fee code: 0004-9729/95 \$A2.00+0.00. 
We are interested in answering the question: Which finite algebras admit a natural duality (are dualisable)? For the definition of admitting a natural duality see [6], or for more details, [4]. It is easy to see that if a finite algebra $\underline{P}$ admits a natural duality then the dualising object can always be chosen to be the above structure $\mathbf{P}$. The following claim which may stand as an alternative definition of dualisable algebras has been proven by Clark and Krauss [2, Theorem 2.25].

THEOREM 1.1. A finite algebra $\underline{P}$ admits a natural duality if and only if for every term-closed set $X \subseteq P^{S}$ every morphism $\alpha: \mathrm{X} \rightarrow \mathrm{P}$ extends to an $S$-ary term operation of $\underline{P}$.

In Section 2, by using Theorem 1.1, we shall present a theorem which gives a better insight into the nature of dualisable algebras. In Section 3, Problem 1 of Davey [6] is partly answered by exhibiting a set of constructs by which the set of all algebraic relations is obtained from any of its entailment-dense subsets. With the help of these constructs we characterise when a finite set of algebraic relations yields a duality for a finite algebra. A familiar theorem in [4] states that $\underline{P}$ admits a natural duality whenever it has a near unanimity term operation. In Section 4 we shall describe a broad class of finite algebras such that in this class each algebra that admits a natural duality necessarily has a near unanimity term operation.

\section{A DUALITY THEOREM}

In quite a few papers dealing with duality theory we find a so called duality theorem that characterises when a finite algebra admits a natural duality. See for example [2, $4,7,8]$. All those theorems are just paraphrasing Theorem 1.1. Nevertheless, Theorem 1.1 somehow is not satisfying when we would like to decide whether a particular finite algebra is dualisable or not. In this section we are going to prove our duality theorem. From this theorem it follows, for example, that in Theorem 1.1, $S$ can be assumed to be countable.

We call a map $\beta: X \rightarrow P, X \subseteq P^{T}$, nonextendible if it does not extend to a $T$-ary term operation of $\underline{P}$. For $H \subseteq T, \pi_{H}$ denotes the projection map from $P^{T}$ to $P^{H}$. There is some ambiguity in this notation when $T$ varies. This will not cause any problem. From the context it will always be clear what $T$ we consider. We also use $\pi_{H}$ instead of $\left.\pi_{H}\right|_{X}$ where $X \subseteq P^{T}$.

THEOREM 2.1. For a finite algebra $\underline{P}$ the following are equivalent.

(1) $\underline{P}$ admits no natural duality.

(2) There exist an increasing sequence of finite sets $S_{i}$, subsets $X_{i} \subseteq P^{S_{i}}$ with $X_{i}=\pi_{S_{i}}\left(X_{i+1}\right)=\pi_{S_{i}}\left(X_{i+1}^{\prime}\right), 0 \leqslant i<\omega$, and a map $\alpha_{0}: X_{0} \rightarrow P$ such that for each $i$ the map $\alpha_{i+1}=\alpha_{i} \circ \pi_{S_{i}}: X_{i+1} \rightarrow P$ preserves all $(i+1)$-ary algebraic relations and is nonextendible. 
(3) There exist an increasing sequence of finite sets $T_{i}$, term-closed sets $Y_{i} \subseteq$ $P^{T_{i}}$ with $\pi_{T_{i}}\left(Y_{i+1}\right) \subseteq Y_{i}, 0 \leqslant i<\omega$, and a map $\beta_{0}: Y_{0} \rightarrow P$ such that for each $i$ the map $\beta_{i+1}=\beta_{i} \circ \pi_{T_{i}}: Y_{i+1} \rightarrow P$ preserves all $(i+1)$-ary algebraic relations and is nonextendible.

Proof: (1) implies (2): Let $\underline{P}$ be a non-dualisable finite algebra. By Theorem 1.1 there exist a term-closed set $X \subseteq P^{S}$ and a morphism $\alpha: X \rightarrow P$ that does not extend to $P^{S}$ as an $S$-ary term operation. Since $\alpha$ is continuous and $X$ is compact there is a finite subset $S_{0} \subseteq S$ such that $\alpha=\alpha_{0} \circ \pi_{S_{0}}$ for some $\alpha_{0}: X_{0} \rightarrow P$, where $X_{0}=\pi_{S_{0}}(X)$. Starting with $S_{0}, X_{0}$ and $\alpha_{0}$ we give a recursive definition of $S_{i}, X_{i}$ and $\alpha_{i}, 0 \leqslant i<\omega$. The $(i+1)$-th step of this is as follows.

Assume that we have already defined $S_{i}, X_{i}$ and $\alpha_{i}$ satisfying the properties in the claim such that $S_{i} \subseteq S$ and $X_{i}=\pi_{S_{i}}(X)$. We are going to define $S_{i+1}, X_{i+1}$ and $\alpha_{i+1}$ such that $S_{i+1} \subseteq S$ and $X_{i}=\pi_{S_{i+1}}(X)$. Let us take an arbitrary $(i+1)$-ary algebraic relation $r$. For every $x_{0}, \ldots, x_{i} \in X$ with $\left(\alpha\left(x_{0}\right), \ldots, \alpha\left(x_{i}\right)\right) \notin r$ there is an $s \in S$ such that $\left(x_{0}(s), \ldots, x_{i}(s)\right) \notin r$. To each $x_{0}, \ldots, x_{i}$ and $s$ of this type we assign an open set of $\left(P^{S}\right)^{i+1}$ of the form $\left\{\left(a_{0}, \ldots, a_{i}\right): a_{j} \in P^{S}\right.$ and $a_{j}(s)=x_{j}(s)$ for each $\left.0 \leqslant j \leqslant i\right\}$. These open sets cover the compact set $\left(\alpha^{i+1}\right)^{-1}\left(P^{i+1} \backslash r\right)$ where $\alpha^{i+1}: X^{i+1} \rightarrow P^{i+1}$ is the continuous map defined by $\alpha^{i+1}\left(x_{0}, \ldots, x_{i}\right)=\left(\alpha\left(x_{0}\right), \ldots, \alpha\left(x_{i}\right)\right)$. So some finitely many of them also do it. Choose such a finite cover for every algebraic relation. Let $H_{i+1}$ be the union of the sets of elements which occur in the definition of the finite cover related to some $(i+1)$-ary algebraic relation. So $H_{i+1} \subseteq S$ is finite. Observe that for every $H$ with $S_{0} \cup H_{i+1} \subseteq H$, the map $\alpha_{0} \circ \pi_{S_{0}}: \pi_{H}(X) \rightarrow P$ preserves all $(i+1)$-ary algebraic relations.

The set $A=\pi_{S_{i}}^{-1}\left(X_{i}^{\prime} \backslash X_{i}\right)$ is closed in $P^{S}$ and $A \cap X=\emptyset$. So for every $a \in A$ there are $S$-ary terms $\sigma_{a}$ and $\tau_{a}$ agreeing on $X$ and differing on $a$. The open sets $\left\{b: b \in P^{S}\right.$ and $\left.\sigma_{a}(b) \neq \tau_{a}(b)\right\}$ cover $A$. Again, some finitely many of them do it. The finitely many term operations occurring in the definition of this finite cover depend on only finitely many variables determining a finite subset $K_{i+1}$ of $S$. Let $S_{i+1}=S_{i} \cup H_{i+1} \cup K_{i+1}, X_{i+1}=\pi_{S_{i+1}}(X)$ and $\alpha_{i+1}=\alpha_{i} \circ \pi_{S_{i}}: X_{i+1} \rightarrow P$.

Let us check the properties mentioned in (2). We have that

$$
X_{i}=\pi_{S_{i}}(X)=\pi_{S_{i}}\left(\pi_{S_{i+1}}(X)\right)=\pi_{S_{i}}\left(X_{i+1}\right)
$$

So $X_{i} \subseteq \pi_{s_{i}}\left(X_{i+1}^{\prime}\right)$. But $\pi_{S_{i}}\left(X_{i+1}^{\prime}\right) \subseteq X_{i}^{\prime}$ and $K_{i+1} \subseteq S_{i+1}$. Hence $X_{i}=\pi_{S_{i}}\left(X_{i+1}^{\prime}\right)$. The map $\alpha_{i}$ is not extendible as $\alpha$ is not and it preserves all $(i+1)$-ary relations since $S_{0} \cup H_{i+1} \subseteq S_{i+1}$.

(2) implies (3): Now, suppose that there is a sequence of $S_{i}$ with $S_{i} \subseteq S_{i+1}$, $X_{i} \subseteq P^{S_{i}}, 0 \leqslant i<\omega$ and $\alpha_{0}: X_{0} \rightarrow P$ as in (2). Let $T_{i}=S_{i+1}$ and $Y_{i}=X_{i+1}^{\prime}$, 
$0 \leqslant i<\omega$. Moreover let $\beta_{0}=\alpha_{0} \circ \pi_{S_{0}}: Y_{0} \rightarrow P$. Clearly, $\pi_{T_{i}}\left(Y_{i+1}\right) \subseteq Y_{i}, 0 \leqslant i<\omega$. Let $\beta_{i+1}=\beta_{i} \circ \pi_{T_{i}}: Y_{i+1} \rightarrow P$. So $\beta_{i+1}=\alpha_{i+1} \circ \pi_{S_{i+1}}: X_{i+2}^{\prime} \rightarrow P$. Since $\alpha_{i+1}$ and $\pi_{S_{i+1}}$ preserve the $i+1$-ary algebraic relations so does $\beta_{i+1}$. Finally, $\beta_{i+1}$ is not extendible since $\alpha_{i+2}$ is not.

(3) implies (1): Let us suppose that (3) holds. We define the countable set $T=$ $\bigcup_{i=0}^{\omega} T_{i}$ and $Y=\bigcap_{i=0}^{\omega} \pi_{T_{i}}^{-1}\left(Y_{i}\right) \subseteq P^{T}$. Then $\pi_{T_{i}}(Y) \subseteq Y_{i}$. Let $\beta=\beta_{0} \circ \pi_{T_{0}}: Y \rightarrow P$. First we show that $Y$ is term-closed. Let $a \notin Y$. Then there exists an $i$ such that $\pi_{T_{i}}(a) \notin Y_{i}$. Since $\pi_{T_{i}}\left(Y_{i+1}\right) \subseteq Y_{i}$ we have that $\pi_{T_{i+1}}(a) \notin Y_{i+1}$. The set $Y_{i+1}$ is term-closed and hence there exist two terms $\sigma$ and $\tau$ agreeing on $Y_{i+1}$ and differing on $\pi_{i+1}(a)$. Since $\pi_{T_{i+1}}(Y) \subseteq Y_{i+1}$, by using the terms $\sigma \circ \pi_{T_{i+1}}$ and $\tau \circ \pi_{T_{i+1}}$ we get $a \notin Y^{\prime}$. Clearly, $\beta$ is well defined, continuous and preserves all algebraic relations. Next, we show that $\beta$ is nonextendible.

First, we prove that for every $i$ there exists a $j>i$ such that $\pi_{T_{i}}(Y)=\pi_{T_{i}}\left(Y_{j}\right)$. For an arbitrary $i$ with $0 \leqslant i<\omega, P^{T_{i}}$ is finite and so there exists a $j$ such that for every integer $k \geqslant j$ the $\pi_{T_{i}}\left(Y_{k}\right)$ are the same. We claim that $\pi_{T_{i}}(Y)=\pi_{T_{i}}\left(Y_{j}\right)$. Without loss of generality we can assume that the sets $T_{i}$ and $T_{k}, j \leqslant k<\omega$, are pairwise different. We define a graph on the set $\pi_{T_{i}}\left(Y_{j}\right) \cup\left(\bigcup_{j \leqslant k<\omega} Y_{k}\right)$ by connecting $a$ and $b$ by an edge if and only if either $a \in Y_{k}, b \in Y_{k+1}$ and $\pi_{T_{k}}(b)=a$ or $a \in \pi_{T_{i}}\left(Y_{j}\right)$, $b \in Y_{j}$ and $\pi_{T_{i}}(b)=a$. By the definition of $j$, for every $a \in \pi_{T_{i}}\left(Y_{j}\right)$ and $k$ with $j \leqslant k<\omega$ there is a path between $a$ and some element of $Y_{k}$. Then, by König's lemma, there is an infinite path starting from $a$ in the graph. This path yields us an element $c \in P^{T}$ such that $\pi_{T_{l}}(c) \in Y_{l}$ for each $l$ with $0 \leqslant l<\omega$, whence $c \in Y$. So for every $a \in \pi_{T_{i}}\left(Y_{j}\right)$ there exists a $c \in Y$ such that $a=\pi_{T_{i}}(c)$. This gives $\pi_{T_{i}}(Y)=\pi_{T_{i}}\left(Y_{j}\right)$.

Now, suppose that $\beta$ is extendible. Then there exists an $i$ such that $\left.\beta_{i}\right|_{\pi_{T_{i}}(Y)}$ is extendible and, as we saw in the preceding paragraph, there exists a $j>i$ such that $\pi_{T_{i}}\left(Y_{j}\right)=\pi_{T_{i}}(Y)$, but this contradicts the fact that $\beta_{j}=\beta_{i} \circ \pi_{T_{i}}: Y_{j} \rightarrow P$ is nonextendible. Hence $\beta$ is nonextendible. So by applying Theorem 1.1 we get (1).

We mention that the argument for constructing $H_{i+1}$ occurs in the proof of Theorem 1.19 in [4].

\section{Entailing algebraic RELations}

In practice for a dualisable finite algebra $\underline{P}$ the structure $\mathbf{P}$ is rarely used as a dualising object. Instead of the set of all algebraic relations a possibly small subset $R$ of it is taken with the property that for every term-closed $X$ every $R$ preserving continuous map of the form $\alpha: X \rightarrow P$ preserves all algebraic relations, that is, $\alpha$ is a morphism from $\mathrm{X}$ to $\mathrm{P}$. For an $R$ with this property we say that $R$ entails the 
set of all algebraic relations, for short, $R$ is an entailment-dense set. In this section we give a characterisation of entailment-dense sets which is similar to the one given by Bodnarčuk, Kalužnin, Kotov and Romov in [1] and by Geiger in [10]. The constructs we need are the following:

diagonal relations, direct product of two relations, intersection of two relations of the same arity, and bijective projection to some variables of a relation.

We mention that with the help of the above set of constructs one can exhibit permutation of variables of a relation, a useful construct that we need later in Theorem 3.3. Also, notice that if we left off the word bijective in (*) we would get the constructs used in [1] and [10].

THEOREM 3.1. For a finite algebra $\underline{P}$ a set $R$ of algebraic relations entails the set of all algebraic relations if and only if every algebraic relation can be obtained from $R$ by using the constructs in (*) in a finite number of steps.

Proof: Let $\underline{P}$ be a finite algebra and let $R$ be a set of algebraic relations of $\underline{P}$. With the exception of bijective projections, in [4] it is mentioned that for every (termclosed) set $X$ every $R$ preserving continuous map $\alpha: X \rightarrow P$ preserves all algebraic relations obtained from $R$ via the constructs in (*). Now let $r \in R$ and let $\pi: r \rightarrow r_{0}$ be a bijective projection. Suppose that $X$ is a term-closed subset of $P^{S}$ for some $S$ and $\alpha: X \rightarrow P$ is an $R$-preserving continuous map. We claim that $\alpha$ preserves $r_{0}$, as well. Let $x_{1}, \ldots, x_{m}$ be some elements of $X$ such that $\left(x_{1}, \ldots, x_{m}\right) \in r_{0}$. By composing any projection to some variable of $r$ with $\pi^{-1}$ we get a homomorphism from $r_{0}$ to $\underline{P}$. Moreover, the hom-closure of $\left\{x_{1}, \ldots, x_{m}\right\}$ is contained in $X$. Hence $X$ contains some elements $y_{1}, \ldots, y_{n}$ with $x_{1}, \ldots, x_{m}$ among them such that $\left(y_{1}, \ldots, y_{n}\right) \in r$. Since $\alpha$ preserves $r$ we have $\left(\alpha\left(y_{1}\right), \ldots, \alpha\left(y_{n}\right)\right) \in r$ and so $\left(\alpha\left(x_{1}\right), \ldots, \alpha\left(x_{m}\right)\right) \in r_{0}$. This gives the "if" part of the theorem.

To prove the "only if" part let us assume that $R$ is an entailment-dense set. Let $H$ denote the set of relations that are obtained from $R$ via (*). Let $r$ be an arbitrary algebraic relation. We shall prove that $r$ is in $H$. Let $X \subseteq P^{r}$ be the set of homomorphisms from $r$ to $\underline{P}$. Then

$$
\neg X=\left\{\left.\pi_{a}\right|_{X}: \pi_{a} \text { is the } a \text {-th projection from } P^{r} \text { to } P, a \in r\right\} .
$$

Hence for every homomorphism $h: \neg X \rightarrow \underline{P}$ we have $h\left(\pi_{a} \mid X\right)=h \circ e(a)$ where $e: r \rightarrow \neg X$ is the isomorphism given by $e(a)=\left.\pi_{a}\right|_{X}$, that is, $h$ is the $h$ o $e$ th projection. Since for every $h, h \circ e \in X$ the set $X$ is hom-closed. Thus $X$ is 
term-closed. Clearly, $r$ can be obtained from $\neg X$ by using a bijective projection and a suitable permutation of variables. So it is sufficient to show that $\neg X \in H$. The set $R$ is entailment-dense, and $X$ is finite and term-closed. Hence every $R$-preserving map from $X$ to $P$ preserves the algebraic relation $\neg X$. Therefore, the set of $R$-preserving maps from $X$ to $P$ coincides with $\neg X$. Let $r_{1}$ be the smallest $X$-ary relation in $H$ containing $\neg X$. If there exists a $\varphi \in r_{1} \backslash \neg X$ then there is a relation not preserved by $\varphi$ in $R$. By intersecting this relation by an appropriate diagonal relation, then by applying a suitable bijective projection we can get an $m$-ary relation $r_{2} \in H$ such that there exist pairwise different elements $y_{1}, \ldots, y_{m} \in X$ with $\left(y_{1}, \ldots, y_{m}\right) \in r_{2}$ and $\left(\varphi\left(y_{1}\right), \ldots, \varphi\left(y_{m}\right)\right) \notin r_{2}$. By taking the direct product of a full diagonal relation and $r_{2}$, then permuting variables we get an $X$-ary relation $r_{3}=\left\{\psi:\left(\psi\left(y_{1}\right), \ldots, \psi\left(y_{m}\right)\right) \in r_{2}\right\} \in H$. Observe that $\neg X \subseteq r_{3}$ and so $\neg X \subseteq r_{1} \cap r_{3}$. Moreover, $r_{1} \cap r_{3} \in H$ and because $\varphi \notin r_{3}, r_{1} \cap r_{3}$ is smaller than $r_{1}$. This contradiction gives $\neg X=r_{1} \in H$.

Recently, in [9] Davey, Haviar and Priestly have got results similar to Theorem 3.1 in a more general context. In fact, in their paper they give a solution to Problem 1 in [6].

We say that a set $R$ of algebraic relations yields a duality for $\underline{P}$ if $\underline{P}$ is dualisable and $R$ entails the set of algebraic relations of $\underline{P}$. In other words, $R$ yields a duality for $\underline{P}$ if for every term-closed set $X \subseteq P^{S}$ every $R$-preserving continuous map $\alpha: X \rightarrow P$ extends to an $S$-ary term operation of $\underline{P}$. Theorem 3.1 gives a necessary condition for a set of algebraic relations to yield a duality for a finite algebra.

Corollary 3.2. Let $\underline{P}$ be a finite algebra. If a set of algebraic relations $R$ yields a duality for $\underline{P}$ then all algebraic relations can be obtained from $R$ via the constructs in $(*)$.

THEOREM 3.3. Let $\underline{P}$ be a finite algebra and let $R$ be a set of algebraic relations of $\underline{P}$. Suppose that $r$ is a relation obtained from $R$ via the constructs in (*). Then

$$
r=\pi\left(\bigcap_{i \in I} \mu_{i}\left(r_{i, 1} \times \cdots \times r_{i, m_{i}}\right)\right)
$$

for some finite set $I$ where the $r_{i, j}$ are elements in $R$ or diagonal relations for all $i \in I$, $1 \leqslant j \leqslant m_{i}, \pi$ is a bijective projection to some variables and the $\mu_{i}$ are permutations of variables for all $i \in I$.

Proof: Let $H$ be the set of relations of the form given in (**). Since the elements of $R$ are of this form we only have to show that $B$ is closed under the constructs in (*). The proof of this is a routine calculation. So we highlight only the closedness under intersection. Suppose that we have an $S$-ary relation $r_{1}$ and a $T$-ary relation $r_{2}$ such that $U=S \cap T$ and $\pi_{U}\left(r_{1}\right)$ and $\pi_{U}\left(r_{2}\right)$ are in $H$ where $\pi_{U}$ is a bijective 
projection in both cases. We define two $(S \cup T)$-ary relations $t_{1}$ and $t_{2}$ as follows. Let $t_{1}=\left\{\varphi:\left.\varphi\right|_{S} \in r_{1}\right\}$ and let $t_{2}=\left\{\varphi:\left.\varphi\right|_{T} \in r_{2}\right\}$. Then $\pi_{U}\left(t_{1} \cap t_{2}\right)=\pi_{U}\left(r_{1}\right) \cap \pi_{U}\left(r_{2}\right)$. Since $\pi_{U}$ is bijective from $t_{1} \cap t_{2}$ to its image, $B$ is closed under intersection.

The following interesting theorem holds.

ThEOREM 3.4. Let $\underline{P}$ be a finite algebra and let $R$ be a finite set of algebraic relations. The following are equivalent.

(1) $R$ yields a duality for $\underline{P}$.

(2) $R$ is an entailment-dense set.

(3) Every algebraic relation is built from $R$ by using constructs in (*).

PROOF: By Theorem 3.1, we get that (2) and (3) are equivalent and (1) implies (2) by definition. So it suffices to show only that (2) implies (1). Let $R$ be a finite entailment-dense set. We shall prove that $\underline{P}$ is dualisable. If $\underline{P}$ is not dualisable then (3) of Theorem 2.1 holds. Since $R$ is finite there exists a positive integer $i$ that is an upper bound for the arities of the relations in $R$. Let us take $Y_{i} \subseteq P^{T_{i}}$ and $\beta_{i}$ as in (3) of Theorem 2.1. Since $R$ is an entailment-dense set and $Y_{i}$ is term-closed all algebraic relations are preserved by $\beta_{i}$. Hence $\beta_{i}$ preserves the finite algebraic relation $\neg Y_{i}$ and so it extends to a $T_{i}$-ary term operation. This contradicts (3) of Theorem 2.1. Thus $R$ yields a duality for $\underline{P}$.

In all known examples for dualisable algebras the duality is yielded by a finite set of algebraic relations. The question whether there exist dualisable algebras of other types remains open. We think that Theorem 2.1 can be a useful tool in answering this question.

Finally, we state a recent result of Willard, [11], that can be obtained as an easy consequence of Theorem 3.4 by using the proof of the "only if" part in Theorem 3.1.

COROLlary 3.5. Let $\underline{P}$ be a finite algebra. If a finite set of algebraic relations $R$ yields a duality on the class of the finite members of the quasi-variety generated by $\underline{P}$ then $R$ yields a duality on the quasi-variety generated by $\underline{P}$.

\section{NATURAL DUALITIES FORCING NEAR UNANIMITY}

This section is separate from the others in the sense that instead of looking for a duality criterion in the class of all finite algebras we examine a smaller class. It will turn out that this class is broad enough to contain some familiar classes of algebras but it is small enough not to have dualisable algebras other than the ones possessing a near unanimity term operation.

For an arbitrary set $S$ let

$N_{S}=\left\{a \in P^{S}:\right.$ for some $p \in P, a(s)=p$ for all $s \in S$ with at most one exception $\}$. 
For each $l \geqslant 2$ let

$C_{l}=\left\{a \in P^{\omega}:\right.$ all but one block of the kernel of $a$ has at most $l-1$ elements $\}$

Let $k$ be a positive integer. For $p$ and $q$ in $P$ let $t_{p, q}$ denote the $2 k$-tuple whose first $k$ components are $p$ and the remaining ones are $q$.

LEMma 4.1. Let $\underline{P}$ be a finite algebra. The following are equivalent.

(1) There is an integer $k \geqslant 2$ such that for all $p$ and $q$ in $P$ with $p \neq q$ there exist $2 k$-ary term operations $\sigma$ and $\tau$ satisfying $\sigma\left(t_{p, q}\right) \neq$ $\tau\left(t_{p, q}\right)$ and $\left.\sigma\right|_{N_{2 k}}=\left.\tau\right|_{N_{2 k}}$.

(2) The term-closure of $N_{\omega}$ is a subset of $C_{l}$ for some $l \geqslant 2$.

Proof: Let $a \in N_{\omega}^{\prime}$. First we show that, if $a \notin C_{k}$ then (1) does not hold. If $a \notin C_{k}$ then there exist different $p$ and $q$ in $P$ such that $k$ components of $a$ equal $p$ and $k$ components of $a$ equal $q$. Since $a$ is in the term closure of $N_{\omega}$ it follows that every pair of terms agreeing on $N_{\omega}$ must agree on $a$. But by adding fictitious variables to the terms guaranteed for $p$ and $q$ by (1) we get two terms agreeing on $N_{\omega}$ and differing on $a$. Hence (1) cannot hold.

Conversely, let us suppose there is some $l$ with $N_{\omega}^{\prime} \subseteq C_{l}$. Let $p$ and $q$ be arbitrary elements of $P$ with $p \neq q$. Let us form the $\omega$-tuple $a=(p, q, p, q, \ldots, p, q, \ldots) \notin C_{l}$. So $a \notin N_{\omega}^{\prime}$. Then there exist two term operations agreeing on $N_{\omega}$ and differing on $a$. Now, there is a finite $k$ such that both term operations depend on their first $2 k$ variables. This $k$ is chosen to be the same for all $p$ and $q$. The $k$ so obtained clearly satisfies the requirements of $(1)$.

The equivalent conditions of Lemma 4.1 hold in quite a large class of finite algebras. In Theorem 4.1 of [7], condition (2) of Lemma 4.1, with $l=2$ has been verified for every finite algebra that generates a variety or a prevariety whose finite members have join semi-distributive congruence or relative congruence lattices, respectively. The following also holds.

ThEOREM 4.2. Let $\underline{P}$ be a finite algebra which generates a variety $V$ whose finite members have join semi-distributive congruence lattices or a prevariety $A$ whose finite members have join semi-distributive $A$-congruence lattices. Then $\underline{P}$ satisfies (1) in Lemma 4.1 for $k=2$.

Proof: Let $\underline{P}$ be a finite algebra generating a prevariety $A$ whose finite members have join semi-distributive $A$-congruence lattices. Let $p$ and $q$ be two different elements in $P$. We show that $(p, p, q, q) \notin N_{4}^{\prime}$. By way of contradiction let us suppose that $(p, p, q, q) \in N_{4}^{\prime}$. This implies that there exists a homomorphism $f: \neg N_{4} \rightarrow \underline{P}$ with $f(a)=f(b)=p$ and $f(c)=f(d)=q$, where $a=\pi_{0}\left|N_{4}, b=\pi_{1}\right|_{N_{4}}, c=\left.\pi_{2}\right|_{N_{4}}$ and $d=\pi_{3} \mid N_{4}$. 
We now define some $A$-congruences on the algebra $\neg N_{4}$. Let $\mu$ be the least $A$ congruence containing the pairs $(a, b)$ and $(c, d)$ and let $\nu$ be the least $A$-congruence identifying $a, b, c$ and $d$. For each $i$ with $1 \leqslant i \leqslant 4$, we define $\theta_{i}$ to be the kernel of the map given by restricting the elements of $\neg N_{4} \subseteq P_{4}^{N}$ to the subset of $N_{4}$ that consists of the 4-tuples with the $i$-th coordinate possibly different from all the others.

Observe that $\bigwedge_{i=1}^{4} \theta_{i}=0$ and $\mu \vee \theta_{i}=\nu, 1 \leqslant i \leqslant 4$, where the lattice operations are taken in the $A$-congruence lattice of $\neg N_{4}$. Since this lattice is join semi-distributive we get $\mu \vee\left(\bigwedge_{i=1}^{4} \theta_{i}\right)=\nu$, that is, $\mu=\nu$. This is impossible since $\mu$ is contained in the kernel of $f$. The same argument, by obvious changes, gives the proof of the claim related to $V$.

It is worth while to mention the following partial converse of Theorem 4.2.

REMARK 4.3. Let $\underline{P}$ be a finite algebra and let $A$ be the prevariety generated by $\underline{P}$. Let us suppose that for every finite $S$ every subset of $P^{S}$ is term-closed. Then the $A$-congruence lattice of every finite member of $A$ is distributive.

Proof: Let $\underline{P}$ be a finite algebra satisfying the condition of the claim. Theorem 4.2 in [3] guarantees us a strong duality between the class of the finite members of $A$ and the class of the finite isomorphic copies of the closed substructures of the powers of P. By the proof of Proposition 1.12 in [5] the $A$-congruence lattice of every finite algebra in $A$ is dually isomorphic to the lattice of closed substructures of its $d u a l$. This dual must also be finite and in our case its lattice of closed substructures is Boolean. So we have the claim.

For example, by the remark, every finite algebra in a prevariety $A$ generated by an order primal algebra has a distributive $A$-congruence lattice.

THEOREM 4.4. Let $\underline{P}$ be a finite algebra. Let us suppose that the equivalent conditions of Lemma 4.1 hold. Then $\underline{P}$ admits a natural duality if and only if it has a near unanimity term operation.

Proof: Let us define a map $\alpha: N_{\omega}^{\prime} \rightarrow P$ by assigning its dominating component to each $a \in N_{\omega}^{\prime}$. Now, $\alpha$ is well defined and continuous since $N_{\omega}^{\prime} \subseteq C_{l}$ for some $l$. Also, $\alpha$ preserves every relation on $N_{\omega}^{\prime}$ since $\alpha$ restricted to any finite subset of $N_{\omega}^{\prime}$ is a projection to some component. So if $\alpha$ does not extend to $P^{\omega}$ via a term operation then by Theorem 1.1, $\underline{P}$ admits no natural duality. If $\alpha$ extends, its extension gives a near unanimity term operation on $\underline{P}$.

\section{REFERENCES}

[1] V.G. Bodnarčuk, L.A. Kalužnin, V.A. Kotov and V.A. Romov, 'Galois theory for Post algebras I, II', (in Russian), Kibernetika (Kiev) 5 (1969), no.3, 1-10, no.5, 1-9. 
[2] D.M. Clark and P.H. Krauss, 'Topological quasi varieties', Acta Sci. Math. 47 (1984), 3-39.

[3] D.M. Clark and B.A. Davey, 'The quest for strong dualities', J. Austral. Math. Soc. (to appear).

[4] B.A. Davey and H. Werner, 'Dualities and equivalences for varieties of algebras', Colloq. Math. Soc. János Bolyai 33 (1983), 101-275.

[5] B.A. Davey and H.A. Priestley, 'Generalized piggyback dualities and applications to Ockham algebras', Houston J. Math. 13 (1987), 151-197.

[6] B.A. Davey, 'Duality theory on ten dollars a Day', in Algebras and orders, (I.G. Rosenberg and G. Sabidussi, Editors), NATO Advanced Study Institute Series, Series C 398 (Kluwer Academic Publishers, 1993), pp. 71-111.

[7] B.A. Davey, L. Heindorf and R. McKenzie, 'Near unanimity: an obstacle to general duality theory', Algebra Universalis (to appear).

[8] B.A. Davey and I.G. Rosenberg, 'Algebraic duality', (preprint).

[9] B.A. Davey, M. Haviar and H.A. Priestley, 'The syntax and semantics of entailment in duality theory', (preprint).

[10] D. Geiger, 'Closed systems of functions and predicates', Pacific J. Math. 27 (1968), 95-100.

[11] R.D. Willard, Personal communication.

JATE

Bolyai Intézet

Aradi Vértanúk Tere 1

H-6720 Szeged

Hungary 